\title{
Cosmological measurement of neutrino mass in the presence of leptonic asymmetry
}

\author{
Julien Lesgourgues ${ }^{1}$, Sergio Pastor ${ }^{1}$ and Simon Prunet ${ }^{2}$ \\ 1 SISSA-ISAS and INFN, Sezione di Trieste, Via Beirut 4, 34014 Trieste, Italy \\ ${ }^{2}$ CITA, 60 St George Street, Toronto, Ontario M5S 3H8, Canada
}

\begin{abstract}
We show that even the smallest neutrino mass consistent with the Super-Kamiokande data is relevant for cosmological models of structure formation and cosmic microwave background (CMB) anisotropies, provided that a relic neutrino asymmetry exists. We calculate the precision with which a $0.07 \mathrm{eV}$ neutrino mass could be extracted from CMB anisotropy and large-scale structure data by the future Planck satellite and Sloan Digital Sky Survey. We find that such a mass can be detected, assuming a large relic neutrino asymmetry still allowed by current experimental data. This measurement of the absolute value of the neutrino mass would be crucial for our understanding of neutrino models.
\end{abstract}

\section{INTRODUCTION}

One of the most intriguing questions in cosmology is the possibility of having an asymmetry in the number of leptons and antileptons in the Universe. This asymmetry is restricted to be in the form of neutrinos from the requirement of universal electric neutrality. A large neutrino asymmetry is not excluded by current observational data of primordial abundances of light elements, cosmic microwave background (CMB) anisotropies and large scale structure in the Universe.

If a relic asymmetry exists, the corresponding neutrinos, called degenerate, are characterized by the dimensionless degeneracy parameter $\xi \equiv \mu / T_{\nu}$, where $\mu$ is their chemical potential and $T_{\nu}$ their temperature. The energy density of degenerate neutrinos is much larger than the one of standard neutrinos, and is a function of $\xi$.

From a theoretical point of view, in most particle physics models the leptonic asymmetry is naturally of the same order as the baryonic one, i.e. one part in $10^{9}-10^{10}$ as required by big bang nucleosynthesis (BBN) [1]. However, there are some specific scenarios where the leptonic asymmetry can grow up to large values in the early universe [2], while the baryonic one remains small. Some examples include lepton asymmetries created by an Affleck-Dine mechanism [3] or by active-sterile neutrino oscillations [4], which allow the neutrino asymmetry to reach order one before neutrinos decouple from the rest of the plasma. In general, these generating mechanisms create a different asymmetry for different neutrino flavors.

From an observational point of view, BBN constrains the neutrino degeneracy to be at most of order one for the electronic neutrino $\left(-0.06 \lesssim \xi_{\nu_{e}} \lesssim 1.1\right)$ [0], but is compatible with larger degeneracies for $\nu_{\mu}$ or $\nu_{\tau}$. Interestingly, the current $\mathrm{CMB}$ anisotropy data are compatible with a large neutrino asymmetry $\xi \sim 3.5$ in the framework of the standard cold dark matter (CDM) cosmological scenario, as shown in [6]. More recently, two of us have shown in a systematic analysis 7 that this conclusion also applies to flat models with a cosmological constant $(\Lambda \mathrm{CDM})$, even when CMB data are combined with constraints on the matter power spectrum (for an earlier discussion see [8]). This analysis is based on the data available when [7] was submitted. We have checked that more recent data such as TOCO [9] and BOOMERANG 97 [10] are still compatible with our previous upper bound, $\xi \lesssim 3.5$.

In the case of massless degenerate neutrinos, the only relevant effect of $\xi$ is to increase the total density of radiation, and to postpone the time of equality between radiation and matter. This modification has got large observable effects: it boosts the first CMB peak amplitude, shifts all peaks to smaller scales, and suppresses matter fluctuations on small scales [7. However, it can be simply described by introducing an effective number of massless neutrino families

$$
N(\xi) \equiv 3+\frac{30}{7}\left(\frac{\xi}{\pi}\right)^{2}+\frac{15}{7}\left(\frac{\xi}{\pi}\right)^{4}
$$

which is as large as 5 for $\xi \simeq 2$. This excess in the effective number of neutrinos would wash out the small corrections that arise in the standard model (slight heating of neutrinos by $e^{+}-e^{-}$annihilations and finite-temperature QED effects), whose effects on the CMB were considered in [11].

The analysis in [7] also included the case of massive degenerate neutrinos, to which we adapted the Boltzmann code CMBFAST by Seljak and Zaldarriaga [12], that calculates the radiation and matter power spectra. It appeared 
that combining the asymmetry with a small mass for one family of neutrinos had some subtle effects, that can not be parametrized simply with $N(\xi)$. For instance, the suppression of small scale matter fluctuations caused by the free-streaming of neutrinos (when they become non-relativistic) is more efficient in presence of an asymmetry, due to the enhanced average momentum of the degenerate neutrino. Also, by combining a mass and a degeneracy for the same neutrino family, we reach a bigger neutrino density today than by introducing these parameters separately, or for different families. This point has very interesting phenomenological consequences.

The evidence for neutrino oscillations from Super-Kamiokande [13], if explained by standard $\nu_{\mu} \leftrightarrow \nu_{\tau}$ oscillations (for recent reviews, see for instance [14]), requires differences of squared masses of the order $\Delta m^{2} \simeq(1-8) \times 10^{-3}$ $\mathrm{eV}^{2}$. This determines a lower limit on the value of the neutrino mass, $m \geq 0.03-0.09 \mathrm{eV}$. This bound is saturated in the case when there is a hierarchy in the neutrino mass pattern, i.e. when the two neutrino masses are very different. In the present work we consider as a typical value $m=m_{S K}=0.07 \mathrm{eV}$.

However such very light neutrinos only make a small contribution to the present energy density of the Universe, of the order $\Omega_{\nu}=0.00075 h^{-2}$ for a dimensionless Hubble parameter $h=H /\left(100 \mathrm{~km} \mathrm{~s}^{-1} \mathrm{Mpc}^{-1}\right)$, while at the same time they have no visible effect on the power spectra of matter and CMB anisotropies. Therefore one concludes that a $0.07 \mathrm{eV}$ neutrino is of little relevance for cosmology. But this conclusion is modified when one considers the combined effects of mass and degeneracy. For instance, the present energy density of neutrinos with $m_{S K}$ and $\xi=3$ is of the same order of magnitude as the one of baryons [6,15]. In such a case the light degenerate neutrino plays the role of a significant hot dark matter component.

The main motivation of this paper is to address the following question: are the future CMB experiments sensitive enough to detect a $0.07 \mathrm{eV}$ neutrino mass in the presence of a relic neutrino asymmetry? Such an evidence would be of tremendous importance for our understanding of neutrino models, since it would probe the absolute value of the neutrino mass, while neutrino oscillations are sensitive to the difference of squared masses. The sensitivity of the future satellite missions [16] Microwave Anisotropy Probe (MAP) and Planck to heavier neutrinos was considered in 117. Since $\xi$ enhances the effect of the mass, but, on the other hand, introduces a new degree of freedom in the model, it is not obvious whether the scheduled experiments have the required sensitivity to detect a degenerate neutrino mass as small as $m_{S K}$. From our calculations we conclude that Planck will be able to detect it, provided that there exists a large relic neutrino asymmetry, typically $\xi \gtrsim 2-3$. This value is close to the one suggested to explain the production of ultra-high energy cosmic rays beyond the Greisen-Zatsepin-Kuzmin cutoff [18].

In a previous work [19], Kinney and Riotto already calculated the precision with which the MAP and Planck satellites could measure a large degeneracy parameter $\xi \sim \mathcal{O}(1)$. The effect that we consider in this work is so tiny that we will skip MAP and focus on the capabilities of Planck, as well as those of the future Sloan Digital Sky Survey (SDSS), that will probe the shape of the matter power spectrum. It is well known that for ordinary massive neutrinos, combining CMB and Large Scale Structure (LSS) data is crucial for the mass extraction [17]; in our case it is even more important, due to the enhanced free streaming effect on small scale matter fluctuations.

For completeness, we also consider the case of a slightly heavier neutrino, with $m=1 \mathrm{eV}$. This mass is of the order of magnitude that could explain the results from the Los Alamos Liquid Scintillation Neutrino Detector (LSND) [20] experiment through neutrino oscillations, which require $\Delta m^{2} \simeq 0.1-1 \mathrm{eV}^{2}$. An eV neutrino mass is also required in cold + hot dark matter models (CHDM), because it produces $\Omega_{\nu} \gtrsim 0.01 h^{-2}$. It has been already shown that such a mass could be extracted with $\sim 20 \%$ precision by Planck + SDSS [17]. We calculate how much this result is improved in the presence of a large asymmetry.

\section{THE FISHER MATRIX}

Since the sensitivity of Planck and of the SDSS is already known, it is possible to assume a "fiducial" model, i.e., a cosmological model that would yield the best fit to the future data, and to forecast the error with which each parameters would be extracted.

Starting with a set of parameters $\theta_{i}$ describing the fiducial model, one can compute the power spectra of CMB temperature and polarization anisotropies. Since the anisotropy data consists in two-dimensional maps of the sky, these power spectra are usually expanded in multipoles $C_{l}^{X}$, where $l$ is the multipole number, and $X$ is one of the temperature or polarization modes $T, E, T E, B$ [21]. Simultaneously, one can derive the linear power spectrum of matter fluctuations $P(k)$, expanded in Fourier space. Although CMB experiments measure the $C_{l}^{X}$ 's directly, redshift surveys such as the SDSS probe the linear power spectrum only on the largest scales, and modulo a biasing factor $b^{2}$. For a given survey, the biasing reflects the discrepancy between the total matter fluctuations in the Universe, and those actually seen by the instruments. It is usually assumed to be independent of $k$.

The error $\delta \theta_{i}$ on each parameter can be calculated from the reduced Fisher matrix $F_{i j}$, which has two terms. The first one accounts for Planck and is computed according to ref. [22], while the second term accounts for the SDSS and 
is calculated following Tegmark 23]

$$
\begin{aligned}
F_{i j} & =\sum_{l=2}^{+\infty} \sum_{X, Y} \frac{\partial C_{l}^{X}}{\partial \ln \theta_{i}} \operatorname{Cov}^{-1}\left(C_{l}^{X}, C_{l}^{Y}\right) \frac{\partial C_{l}^{Y}}{\partial \ln \theta_{j}} \\
& +2 \pi \int_{0}^{k_{\max }} \frac{\partial \ln P_{o b s}(k)}{\partial \ln \theta_{i}} \frac{\partial \ln P_{o b s}(k)}{\partial \ln \theta_{j}} w(k) d \ln k .
\end{aligned}
$$

Here $\operatorname{Cov}\left(C_{l}^{X}, C_{l}^{Y}\right)$ is the covariance matrix of the estimators of the CMB spectra for Planck, and $w(k)$ is the weight function for the bright red galaxies sample of the SDSS, taken from Tegmark [23]. We defined $P_{o b s}(k) \equiv b^{2} P(k)$, and $k_{\max }$ is the maximal wave number on which linear predictions are reliable. Following [17], we will use either the

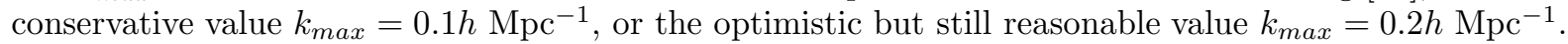

Inverting $F_{i j}$, one obtains the 1- $\sigma$ error on each parameter, assuming that all other parameters are unknown

$$
\frac{\delta \theta_{i}}{\theta_{i}}=\left(F^{-1}\right)_{i i}^{1 / 2} .
$$

It is also useful to compute the eigenvectors of the reduced Fisher matrix (i.e., the axes of the likelihood ellipsoid in the space of relative errors). The error on each eigenvector is given by the inverse square root of the corresponding eigenvalue. The eigenvectors with large errors indicate directions of parameter degeneracy; those with the smallest errors are the best constrained combinations of parameters.

We assume that a best fit to the future Planck and SDSS data (our "fiducial" model) is a $\Lambda$ CDM model with ten parameters: (1) a neutrino mass $m=0,0.07$ or $1 \mathrm{eV},(2)$ a neutrino degeneracy $\xi,(3)$ a Hubble parameter $h=0.65$, (4) a baryon density $\Omega_{b}=0.015 h^{-2}$, (5) a cold dark matter density $\Omega_{C D M}=0.3$, (6) a primordial spectrum tilt $n=0.98$, (7) a primordial spectrum normalization, fixed in CMBFAST by fitting to COBE, (8) an optical depth to reionization $\tau=0.05,(9)$ a quadrupole tensor-to-scalar ratio $T / S=0.14,(10)$ an arbitrary SDSS bias $b$. These parameters were chosen in such way that for $m_{S K}$ and $0 \leq \xi \leq 3.5$, the fiducial models pass the observational tests of [7]. These tests are independent of the bias, and so is the Fisher matrix as can be seen from Eq. (2).

\section{MEASURING THE DEGENERACY PARAMETER}

We first consider a fiducial model with $m=0$ and a degeneracy parameter $\xi$. Our results are shown in Fig. 1, where we plot $\delta \xi / \xi$ as a function of $\xi$. For a very large degeneracy $\xi=3.5$, we find for Planck alone, without polarization, $\delta \xi / \xi=2.5 \%$. Such a small error is justified by the large effect of the degeneracy on the amplitude and shape of the acoustic peaks [6,7,19]. It is limited by a small parameter degeneracy between $\xi$ and $\Omega_{C D M}$. Computing the eigenvectors of the Fisher matrix, we find that the combination $\Omega_{C D M}^{0.8} / \xi^{0.6}$ is measured with $2.9 \%$ uncertainty. This is equivalent to a degeneracy between $\xi$ and the cosmological constant, since when $m=0$, one has that $\Omega_{C D M}+\Omega_{\Lambda}=1-\Omega_{b}$. There is a simple physical explanation: the only effect of $\xi$ is to change the time of equality, and this is one of the main effects of $\Omega_{\Lambda}$. Since this explanation holds not only for the temperature spectrum, but also for the polarization and matter spectra, we do not expect to remove this degeneracy by including the information from polarization and the SDSS: indeed, $\delta \xi / \xi$ is reduced only from $2.5 \%$ to $2.3 \%$. So, only direct precise measurements of $\Omega_{C D M}$ and $\Omega_{\Lambda}$ (using gravitational lensing or supernovae) could improve this already good result.

Our results are in good agreement with Kinney and Riotto [19]. Interestingly, they are found to be almost independent of the mass of the degenerate neutrino. With a significant value of the mass, one may expect to lose precision on $\xi$, due to possible degeneracies between $\xi$ and $m$ : both parameters boost the acoustic peaks and suppress power on small scales. However, the two effects should remain separable, because $\xi$ changes the radiation density at all times, while $m$ affects it only when the neutrinos become non-relativistic. Indeed, for $m=1 \mathrm{eV}$, we check by diagonalizing the Fisher matrix that no degeneracy appears between $\xi$ and $m$. By varying $m$ from 0 to $1 \mathrm{eV}$, we find that $\delta \xi / \xi$ increases by only $\sim 10 \%$.

\section{MEASURING THE DEGENERATE NEUTRINO MASS}

We introduce one family of massive degenerate neutrinos with $m_{S K}$. In this case, the results for $\delta m / m$ as a function

of $\xi$ are shown as the upper curves in Fig. 2. For instance, when $\xi=2.75$, the temperature anisotropy measurement by Planck can bring evidence for $m_{S K}$, but only marginally: $\delta m / m=102 \%$. Here the precision is limited by a 
parameter degeneracy direction $(T / S)^{0.7} / m^{0.7}$, with eigenvalue $130 \%$. Since the polarization measurement is able to constrain $T / S$ better, when including it we find $\delta m / m=93 \%$. Finally, significant progress is made with the SDSS, and the final error depends on $k_{\max }$, since the SDSS is sensitive on small scales to the free streaming produced by

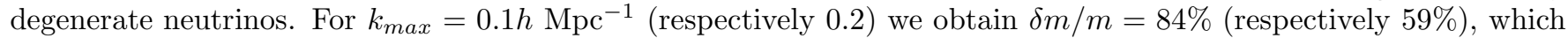
results in a clear detection, especially if we recall that $\partial C_{l} / \partial m$ is a strongly non-linear function of $m$ when $m \rightarrow 0$, so that the above errors, when large, are overestimated, as pointed out in [17]. When the neutrino degeneracy is as large as $\xi=3.5$, we get $\delta m / m=37 \%$ for Planck + SDSS.

We also plot in Fig. 2 the results for $m=1 \mathrm{eV}$ (lower curves). When $\xi \rightarrow 0$, the estimated errors are consistent with [17]. In the presence of a relic neutrino asymmetry, they are lowered from $\delta m / m=15-20 \%$ (for $\xi=0$ ) to $3-4 \%$ (for $\xi=3$ ), depending on $k_{\max }$.

\section{CONCLUSIONS}

We have shown that Planck will be able to detect a neutrino mass of the order $m_{S K}$, provided that the relic neutrinos are strongly degenerate, with a degeneracy parameter $\xi \gtrsim 3$. The combination of Planck with the SDSS improves the results and allows a detection of $m_{S K}$ if $\xi \gtrsim 2.5$. Therefore the neutrino mass suggested by Super-Kamiokande could be relevant for cosmological models of structure formation and CMB anisotropies.

We have also confirmed that in the massless neutrino case, the degeneracy parameter $\xi$ can be extracted from the CMB data by Planck with the precision found by Kinney and Riotto [19]. For this parameter, we find that the inclusion of the SDSS data or the addition of a neutrino mass would not significantly change the results. Last but not least, if the mass of the neutrinos is of the order of $1 \mathrm{eV}$, then even in absence of asymmetry it can be extracted with Planck and SDSS with the precision found by [17], but the relic neutrino asymmetry allows a more accurate detection.

The possibility of detecting a neutrino mass and/or a relic neutrino asymmetry from future cosmological experiments is an example of the fascinating connection between large scale cosmology and particle physics.

\section{ACKNOWLEDGMENTS}

J. Lesgourgues and S. Pastor are supported by the European Commission under the TMR contract ERBFMRXCT960090.

[1] See, for instance, S. Sarkar, Rept. Prog. Phys. 59, 1493 (1996) and references therein.

[2] A. Casas, W.Y. Cheng and G. Gelmini, Nucl. Phys. B538, 297 (1999); J. McDonald, Phys. Rev. Lett. 84, 4798 (2000).

[3] I. Affleck and M. Dine, Nucl. Phys. B249, 361 (1985).

[4] R. Foot, M.J. Thomson and R.R. Volkas, Phys. Rev. D 53, 5349 (1996)

[5] H. Kang and G. Steigman, Nucl. Phys. B372, 494 (1992).

[6] J.A. Adams and S. Sarkar, Report No. OUTP-98-70P and talk presented at the workshop on The Physics of Relic Neutrinos, Trieste, 1998.

[7] J. Lesgourgues and S. Pastor, Phys. Rev. D 60, 103521 (1999).

[8] G.B. Larsen and J. Madsen, Phys. Rev. D 52, 4282 (1995).

[9] A.D. Miller et al., Astrophys. J. 524, L1 (1999).

[10] P.D. Mauskopf et al. [BOOMERANG collaboration], astro-ph/9911444.

[11] R.E. Lopez, S. Dodelson, A. Heckler and M.S. Turner, Phys. Rev. Lett. 82, 3952 (1999).

[12] U. Seljak and M. Zaldarriaga, Astrophys. J. 469, 437 (1996).

[13] Y. Fukuda et al. [Super-Kamiokande Collaboration], Phys. Rev. Lett. 81, 1562 (1998).

[14] J.W.F. Valle, hep-ph/9911224; R.N. Mohapatra, hep-ph/9910365; A.Yu. Smirnov, hep-ph/9907296.

[15] P.B. Pal and K. Kar, Phys. Lett. B451, 136 (1999).

[16] See http://map.gsfc.nasa.gov for information on MAP and http://astro.estec.esa.nl/Planck for Planck.

[17] D.J. Eisenstein, W. Hu and M. Tegmark, Astrophys. J. 518, 2 (1999).

[18] G. Gelmini and A. Kusenko, Phys. Rev. Lett. 82, 5202 (1999).

[19] W.H. Kinney and A. Riotto, Phys. Rev. Lett. 83, 3366 (1999). 
[20] C. Athanassopoulos et al. [LSND Collaboration], Phys. Rev. Lett. 81, 1774 (1998); ibid. 77, 3082 (1996).

[21] M. Kamionkowski, A. Kosowsky and A. Stebbins, Phys. Rev. Lett. 78, 2058 (1997); Phys. Rev. D 55, 7368 (1997); M. Zaldarriaga and U. Seljak, ibid. 55, 1830 (1997).

[22] G. Jungman, M. Kamionkowski, A. Kosowsky and D. L. Spergel, Phys. Rev. Lett. 76, 1007 (1996); Phys. Rev. D 54, 1332 (1996); M. Zaldarriaga, D. N. Spergel and U. Seljak, Astrophys. J. 488, 1 (1997); M. Tegmark, A. Taylor and A. Heavens, ibid. 480, 22 (1997); J. R. Bond, G. Efstathiou and M. Tegmark, Mon. Not. R. Astron. Soc. 291, L33 (1997).

[23] M. Tegmark, Phys. Rev. Lett. 79, 3806 (1997). 
FIG. $1 . \delta \xi / \xi$ versus $\xi$, for one family of massless degenerate neutrinos. From top to bottom, the four curves refer to Planck without and with polarization, and Planck + SDSS with $k_{\max }=0.1$ or $0.2 \mathrm{~h} \mathrm{Mpc}^{-1}$. The results are almost identical in the four cases, showing that the CMB temperature measurement is sufficient to constrain $\xi$.

FIG. 2. $\delta m / m$ versus $\xi$, for one family of massive degenerate neutrinos. The upper curves refer to $m=0.07 \mathrm{eV}$, the lower curves for $m=1 \mathrm{eV}$. In each case, we give the results, from top to bottom, for Planck without and with polarization, and Planck + SDSS with $k_{\max }=0.1$ or $0.2 h \mathrm{Mpc}^{-1}$. 


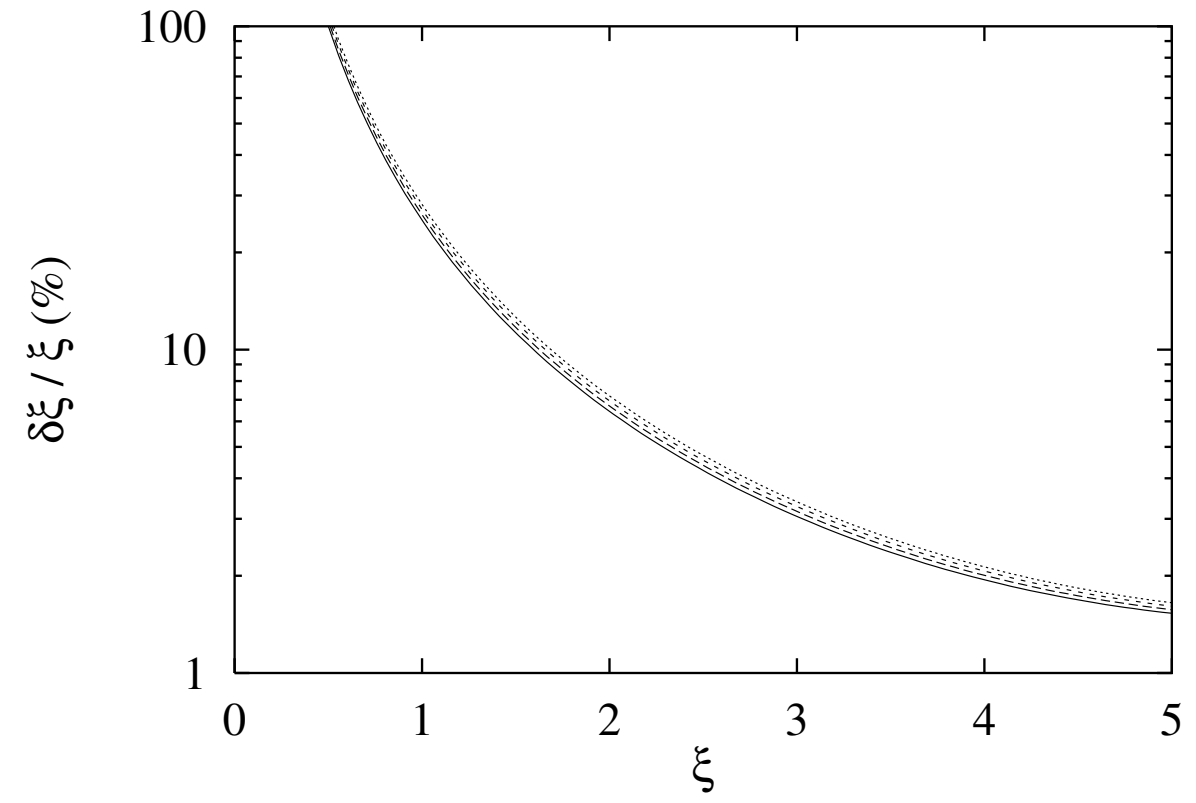

Figure 1 


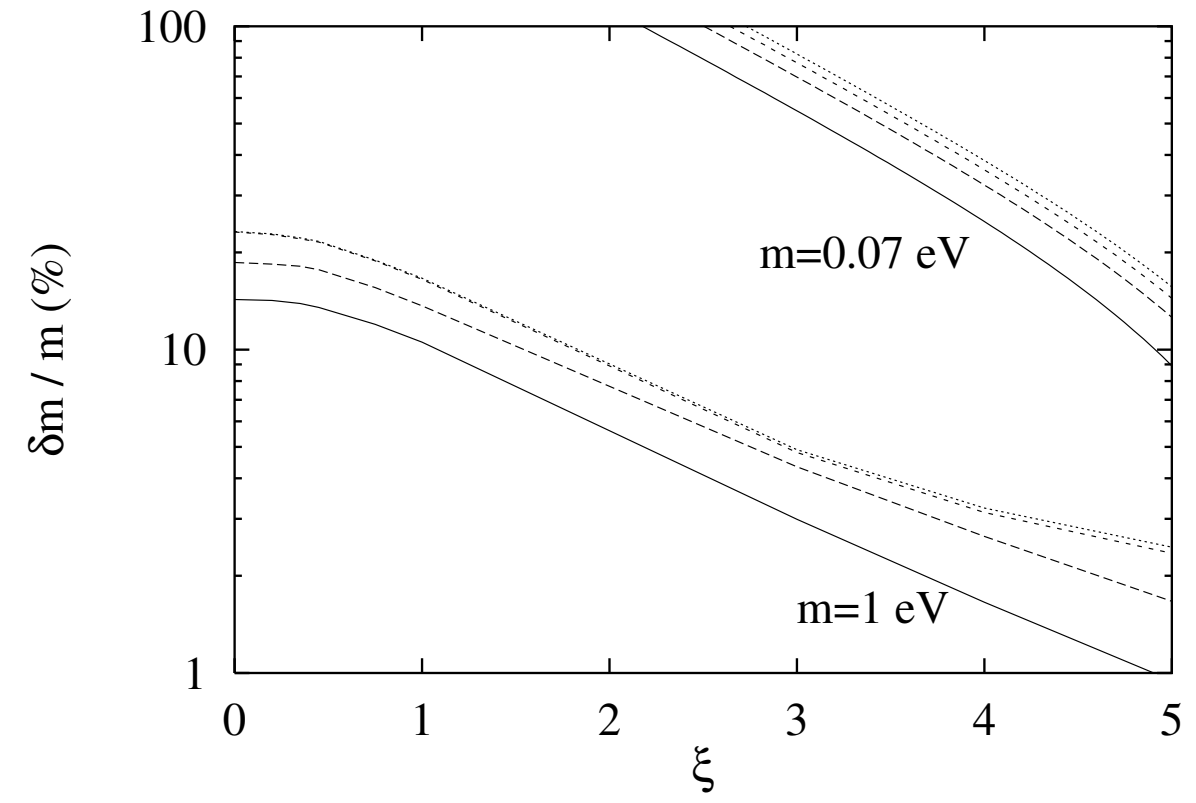

Figure 2 